\title{
If in doubt [Preoperative evaluation of non-small cell lung cancer]...
}

Michael A. Maddaus, MD

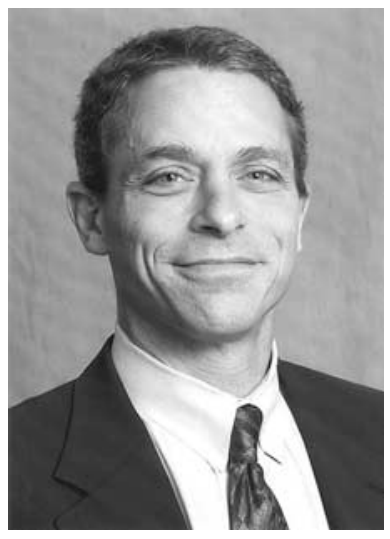

From the Department of Surgery, University of Minnesota, Minneapolis, Minn.

Received for publication Aug 8, 2001; accepted for publication Sept 13, 2001.

Address for reprints: Michael Maddaus, MD, Department of Surgery, University of Minnesota, 420 Delaware St, Box 207, Minneapolis, MN 55455.

J Thorac Cardiovasc Surg 2003;125:S96-7 Originally published in $\mathrm{J}$ Thorac Cardiovasc Surg 2001;122:849-50.

Copyright (C) 2003 by The American Association for Thoracic Surgery

$0022-5223 / 2003 \$ 30.00+0$

doi: $10.1067 / \mathrm{mtc} .2003 .242$

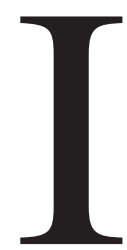
$\mathrm{f}$ in doubt, give the patient the benefit of the doubt." Dr Robert Ginsberg taught me this while I trained with him in the early 1990s at Memorial Sloan-Kettering Cancer Center. Since that moment, I have adhered to this principle, with frequent significant benefit to my patients.

Noninvasive radiologic preoperative staging of non-small cell lung cancer (NSCLC) has dramatically changed over the past decade. High-resolution spiral computed tomography (CT) can now delineate nearly all enlarged hilar and mediastinal lymph nodes and can detect other lung nodules as small as $1.0 \mathrm{~mm}$. Positron emission tomography (PET) scanning now provides a whole-body image search for metastases. Whether CT or PET is used, a problem arises when a result is equivocal or uncertain. With PET scanning, the answer to a negative result may be easy-operate. A positive result, however, may open a Pandora's box of clinical interpretations, ranging from obviously positive (eg, a definite hot spot on a rib), to a faint smudge of positivity (with the surgeon asking the radiologist, "Do you think there is anything really there?"), to errors of interpretation (eg, kidney, renal collecting ducts, bladder, liver). This frequent haze of uncertainty is a regular event with radiologic results.

The article by Buccheri and Ferrigno ${ }^{1}$ attempts to improve on the accuracy of preoperative radiologic evaluation with a simple serum test for two tumor markers: tissue polypeptide antigen (TPA) and carcinoembryonic antigen (CEA). TPA, a member of the cytokeratin family, consists of proteolytic fragments of the cytokeratins 8,18 , and 19. Because TPA is made up of cytoskeleton fragments, elevated levels tend to represent increased cell division and turnover of a tumor. Hypothetically, larger, more advanced tumors may have higher cell division and death rates, with correspondingly higher levels of serum TPA. CEA, a cell-surface glycoprotein of the immunoglobulin gene superfamily, is normally expressed in secretory tissues. CEA may be important for intercellular adhesion and attachment; through those functions it is thought to be potentially involved in tumor cell metastasis.

In the study by Buccheri and Ferrigno, 124 consecutive patients-whose NSCLC had been staged primarily by chest, abdominal, and brain CT (with no PET and only rare mediastinoscopy)—underwent surgical resection. Preoperative serum TPA and CEA levels were correlated with surgical resectability, defined as "pathologic postoperative documentation of stage Ia through IIb disease." The independent accuracy of CT (chest, abdominal, brain) in predicting surgical resectability was $77 \%$; that of TPA levels, 65\%; and that of CEA levels, 69\%. Low levels of TPA $(<90 \mathrm{U} / \mathrm{L})$ and CEA $(<10 \mathrm{ng} / \mathrm{mL})$ increased the accuracy of CT to $83 \%$ and $85 \%$, respectively. Conversely, elevated levels of TPA and CEA increased the accuracy of CT to $89 \%$ and $100 \%$.

Buccheri and Ferrigno defined a cutoff for resectability at the IIb to IIIa point. This cutoff represents relatively advanced disease, most of which would now be detected with PET scanning and mediastinoscopy. Although serum measurements of various tumor markers such as TPA and CEA may incrementally improve staging accuracy, particularly with more advanced disease, a gray zone of uncertainty still remains that can be dispelled only by tissue staging.

The search for a more accurate means of preoperative staging is entering a new and exciting phase, ushered in by advances in molecular biology. With PCR techniques, occult micrometastatic tumor cells can now be detected in histologically 
normal lymph nodes, bone marrow, or blood. In addition, with quantitative PCR, the number of occult micrometastatic tumor cells in a given tissue sample can now be quantitated. Two current trials, the Cancer and Leukemia Group B 9761 and the American College of Surgeons Oncology Group, are now in the process of evaluating the detection of occult micrometastases in patients with NSCLC. The results of these two trials will shed light on the predictive value of such molecular techniques. The ability to preoperatively determine a patient's micrometastatic tumor burden would fundamentally alter preoperative evaluation and treatment planning.

About $50 \%$ of patients with lung cancer now die within
5 years after curative-intent resection. Clearly, our current ability to detect unfavorable tumor biology and disseminated malignant disease is limited. Until molecular-based techniques are routinely available, which will occur, the onus is still on the thoracic surgeon to give the patient the benefit of the doubt.

\section{Reference}

1. Buccheri G, Ferrigno D. Serum biomarkers facilitate the recognition of early-stage cancer and may guide the selection of surgical candidates: a study of carcinoembryonic antigen and tissue polypeptide antigen in patients with operable non-small cell lung cancer. J Thorac Cardiovasc Surg. 2001;122:891-9. 